\title{
I-Love relation for incompressible stars and realistic stars
}

\author{
T. K. Chan*, AtMa P. O. Chan ${ }^{\dagger}$ and P. T. Leung ${ }^{\ddagger}$ \\ Physics Department and Institute of Theoretical Physics, \\ The Chinese University of Hong Kong, Shatin, Hong Kong SAR, China.
}

(Dated: June 23, 2021)

\begin{abstract}
In spite of the diversity in the equations of state of nuclear matter, the recently discovered ILove-Q relations [Yagi and Yunes, Science 341, 365 (2013)], which relate the moment of inertia, tidal Love number (deformability) and the spin-induced quadrupole moment of compact stars, hold for various kinds of realistic neutron stars and quark stars. While the physical origin of such universality is still a current issue, the observation that the I-Love-Q relations of incompressible stars can well approximate those of realistic compact stars hints at a new direction to approach the problem. In this paper, by establishing recursive post-Minkowskian expansion for the moment of inertia and the tidal deformability of incompressible stars, we analytically derive the I-Love relation for incompressible stars and show that the so obtained formula can be used to accurately predict the behavior of realistic compact stars from the Newtonian limit to the maximum mass limit.
\end{abstract}

PACS numbers: 04.40.Dg, 04.25.Nx, 97.60.Gb, 95.30.Sf

\section{INTRODUCTION}

Recently, Yagi and Yunes [1, 2] have discovered the so called "I-Love-Q universal relations" prevailing in compact stars, including both neutron stars (NSs) or quark stars (QSs). In such relations, the moment of inertia $I$, the quadrupole tidal Love number $\lambda$ (or, more precisely, tidal deformability [3, 4] ), and the spin-induced quadrupole moment $Q$ of compact stars are expressed in terms of one another, with the stellar mass $M$ playing the role of a scaling parameter. Soon after the discovery of Yagi and Yunes [1, 2], the I-Love-Q relations were generalized to several other cases, including binary systems with strong dynamical tidal field [5], magnetized NSs with sufficiently high rotation rates 6], rapidly-rotating stars [7, 8], and higher-order multipole moments induced by either tidal forces or rotation [4, 9, 10].

These relations are useful for several reasons [1, 2]. First, they provide a link directly connecting the I-Love-Q triplet. Once the mass of a compact star is known, each one of of $I, \lambda$ and $Q$ can lead to the determination of the other two. Second, in the analysis of gravitational wave signals emitted during the late stages of NS-NS binary mergers, they can break the degeneracy between the contributions due to the quadrupole moment and the spin and hence enable more accurate measurement of the averaged spin of the system [1, 2, 11, 12]. Third, they can identify the validity of other modified gravity theories such as the Chern-Simons gravity and the Eddington-inspired Born-Infeld (EiBI) gravity [1, 2, 13, 14].

On the other hand, the emergence of the I-Love-Q relations is also interesting from theoretical point of view. As is well known, the physical characteristics of NSs (or QSs), including mass, radius, and moment of inertia, are usually obscured by various uncertainties in the equation of state (EOS) of nuclear matter (or quark matter). In fact, nuclear physicists have been using different characteristics (e.g., the mass-radius relation, the maximum mass and gravitational wave spectrum) of NSs (or QSs) as the test bed of various EOSs of dense matter (see, e.g., [15 23]). Nonetheless, the I-Love-Q relations are shown to hold for different commonly accepted EOSs for both NSs and QSs [1, 2, 4]. What is the physical origin of such universal behavior? Yagi and Yunes [1, 2] have suggested two possible reasons for such universality: (i) the relations are mainly dominated by the low-density matter lying in an layer between $70 \%$ and $90 \%$ of the stellar radius where the EOS is well known; and (ii) NSs merely follow the behavior of black-holes and obey the no-hair theorem in the high-compactness limit. However, more recent investigation showed that the I-Love-Q relations are dominated by a thicker layer which is bounded between $50 \%$ and $90 \%$ of the radius and comprises both high and low-density matter [24]. As a result, it is rather unlikely that the similarity of EOSs in the low-density regime can give rise to the I-Love-Q universality.

Moreover, it is interesting to note that the I-Love-Q relations are valid for incompressible stars and QSs [4, 25],

\footnotetext{
* Present address: Department of Physics, University of California at San Diego, 9500 Gilman Drive, La Jolla, CA 92093, USA. Email address: chantsangkeung@gmail.com

$\dagger$ Present address: Department of Physics, University of Illinois at Urbana-Champaign, Urbana, IL 61801-3080, USA. Email address: atma.pochan@gmail.com

‡ Email: ptleung@phy.cuhk.edu.hk
} 
which are constructed from EOSs completely different from those of normal NSs. In particular, Sham et al. [25] have attributed the I-Love-Q universality to (i) the high stiffness of nuclear matter and quark matter, and (ii) the I-Love-Q relations are stationary with respect to variations of stiffness of stellar matter about the incompressible limit. As a result, except very close to the low mass limit of NSs, where NSs are made of relatively soft low-density substance, the I-Love-Q relations of realistic NSs are well approximated by those of incompressible stars to less than a few percent.

The main objective of the present paper is to derive analytically the I-Love relation for incompressible stars. As discovered by Sham et al. [25] and mentioned above, the relation so obtained is also applicable to realistic NSs. We note that, to our knowledge, so far the general relativistic I-Love-Q relations for realistic NSs are only expressed in terms of the empirical formulas suggested by Yagi and Yunes [1, 2] as follows:

$$
\ln y_{i}=a_{i}+b_{i} \ln x_{i}+c_{i}\left(\ln x_{i}\right)^{2}+d_{i}\left(\ln x_{i}\right)^{3}+e_{i}\left(\ln x_{i}\right)^{4},
$$

where $x_{i}$ and $y_{i}$ are any two of the I-Love-Q triplet, $a_{i}, b_{i}, c_{i}, d_{i}$, and $e_{i}$ are some fitting coefficients (see [1, 2] for the values of the these coefficients). By establishing recursive post-Minkowskian perturbative schemes for the (scaled) moment of inertia and tidal Love number (deformability) of incompressible stars, we obtain simple yet accurate formulas expressing these two physical quantities as functions (e.g., power series and fractions) of the stellar compactness. After eliminating the stellar compactness from the formulas, we find the I-Love relation for incompressible stars, which is expressible in terms of simple algebraic functions (see (5.2) and (5.3)). Most importantly, we show that the so derived I-Love relation is also valid for realistic compact stars (including NSs and QSs) and applies to both relativistic and Newtonian stars with high degree of accuracy.

The paper is organized as follows. In Section \we briefly review the solution of the Tolman-Oppenheimer-Volkov (TOV) equations [26, 27], which govern the hydrostatic equilibrium of relativistic stars, for incompressible stars [28] and expand relevant physical quantities (e.g., pressure and metric coefficients) in power series of compactness. In Sections III and IV we formulate recursive perturbative expansions for the moment of inertia and the tidal Love number (deformability) for incompressible stars, respectively. In Section $\nabla$ we find analytic formulas (5.2) and (5.3) relating the moment of inertia and the Love number for incompressible stars and show that these formulas also work nicely for realistic NSs and QSs. We conclude our paper in Section VI with some discussions. Unless otherwise stated explicitly, geometric units in which $G=c=1$ are adopted.

\section{HYDROSTATIC EQUILIBRIUM OF INCOMPRESSIBLE STARS}

Here we briefly review the hydrostatic equilibrium solution of a relativistic, non-rotating compact star made of perfect fluid with a constant density $\rho_{c}$. Since the system is stationary and spherically symmetric, the spacetime metric admits the simple form

$$
d s^{2}=-e^{\nu} d t^{2}+e^{\lambda} d r^{2}+r^{2}\left(d \theta^{2}+\sin ^{2} \theta d \phi^{2}\right),
$$

where $t, r, \theta, \phi$ are the standard Schwarzschild coordinates, $\nu$ and $\lambda$ are some functions of $r$ (see, e.g., [29, 30]). In particular, the metric outside the star is given by the Schwarzschild metric with $e^{\nu}=e^{-\lambda}=1-2 M / r$, where $M$ is the total mass of the star. Inside the star, the TOV equations govern the variation of the pressure $p$ and the metric coefficient $e^{\nu}[26,27]$ :

$$
\begin{aligned}
& \frac{d p}{d r}=-\frac{\left(m+4 \pi r^{3} p\right)\left(\rho_{c}+p\right)}{r^{2}(1-2 m / r)}, \\
& \frac{d \nu}{d r}=-\frac{2}{\rho+p} \frac{d p}{d r},
\end{aligned}
$$

where $m(r)=4 \pi \rho_{c} r^{3} / 3$ is just the gravitational mass enclosed within radius $r$ [26, 27]. The other metric coefficient $e^{\lambda}$ can be determined from $m(r)$ by $e^{-\lambda}=1-2 m(r) / r$. For an incompressible star with a given radius $R$, the TOV equations together with the two boundary conditions (i) $p(R)=0$ and (ii) $e^{\nu(R)}=1-2 M / R$ completely specify both $p(r)$ and $e^{\nu(r)}$ inside the star.

The analytical solution of the TOV equations, (2.2) and (2.3), for incompressible stars, which is known as the Schwarzchild constant-density interior solution [28], is given by:

$$
\begin{aligned}
p(r) & =\rho_{c}\left(\frac{\sqrt{1-2 M r^{2} / R^{3}}-\sqrt{1-2 M / R}}{3 \sqrt{1-2 M / R}-\sqrt{1-2 M r^{2} / R^{3}}}\right), \\
e^{\nu(r)} & =\left(\frac{3}{2} \sqrt{1-\frac{2 M}{R}}-\frac{1}{2} \sqrt{1-\frac{2 M r^{2}}{R^{3}}}\right)^{2},
\end{aligned}
$$


where $r \leq R$. In order to keep the pressure positive and hence physically acceptable, the compactness $\mathcal{C} \equiv M / R$ of incompressible stars must be less than $4 / 9$.

It can be readily shown from (2.4) and (2.5) that both $p(r)$ and $e^{\nu(r)}$ can be considered as functions of the scaled radius $x \equiv r / R$ and the compactness $\mathcal{C}$. Both of them can be expanded as Taylor series in $\mathcal{C}$,

$$
\begin{aligned}
p(x ; \mathcal{C}) & =p_{0}(x)+p_{1}(x) \mathcal{C}+p_{2}(x) \mathcal{C}^{2}+\cdots \\
e^{\nu}(x ; \mathcal{C}) & =\left(e^{\nu}\right)_{0}(x)+\left(e^{\nu}\right)_{1}(x) \mathcal{C}+\left(e^{\nu}\right)_{2}(x) \mathcal{C}^{2}+\cdots
\end{aligned}
$$

where $p_{n}(x)$ and $\left(e^{\nu}\right)_{n}(x), n=0,1,2, \cdots$, are functions of $x$ only. The leading expansions are given explicitly by

$$
\begin{aligned}
p(x ; \mathcal{C}) & =\frac{1}{2} \rho_{c}\left(1-x^{2}\right) \mathcal{C}+\rho_{c}\left(1-x^{2}\right) \mathcal{C}^{2}+\cdots \\
e^{\nu}(x ; \mathcal{C}) & =1-\left(3-x^{2}\right) \mathcal{C}+\frac{3}{4}\left(1-x^{2}\right)^{2} \mathcal{C}^{2}+\cdots
\end{aligned}
$$

For reference and illustration, we tabulate $\bar{p}_{n}(x) \equiv p_{n}(x) / \rho_{c}$ and $\left(e^{\nu}\right)_{n}(x)$ for $0 \leq n \leq 6$ in Tables $\Pi$ and $\coprod$ On the other hand, it is straightforward to show that

$$
\begin{aligned}
e^{\lambda}(x ; \mathcal{C}) & =\frac{1}{1-2 \mathcal{C} x^{2}} \\
& =1+2 \mathcal{C} x^{2}+\left(2 \mathcal{C} x^{2}\right)^{2}+\cdots
\end{aligned}
$$

In the following discussion, we will use the post-Minkowsian expansions in (2.8), (2.9) and (2.10) as the input to evaluate the moment of inertia and the tidal Love number (deformability) of incompressible stars.

\section{MOMENT OF INERTIA}

From the analytic stellar profile derived above, in this section we formulate a post-Minkowskian recursive perturbation scheme to calculate the moment of inertia $I$ of incompressible stars in the slowly rotating limit [31 33]. Consider a uniformly rotating star with a unit angular velocity. Let $\Lambda$ be the angular velocity of the local inertial frame due to the frame-dragging effect of the rotating star [31, 32]. It satisfies the differential equation [31, 32]:

$$
\frac{d}{d x}\left(x^{4} j \frac{d \Lambda}{d x}\right)+4 x^{3} \frac{d j}{d x}(\Lambda-1)=0
$$

where $j(x)=e^{-(\lambda+\nu) / 2}$ for $0 \leq x \leq 1$ and $j(x)=1$ for $x>1$. Outside the rotating star, the above equation can be readily integrated to yield the result $\Lambda=2 I / r^{3}[31,32]$. In particular, the surface value of $\Lambda, \hat{\Lambda} \equiv \Lambda(x=1)$, is equal to $2 I / R^{3}$. Thus, the geometric factor $a \equiv I /\left(M R^{2}\right)$ is given by $a=\hat{\Lambda} / 2 \mathcal{C}$. It is obvious that for incompressible stars $a=2 / 5$ in the Newtonian limit. However, general relativistic effect could lead to modification in the value of $a$.

To solve (3.1) inside the star, similar to what we have done in Section II, we expand $\Lambda(x)$ and $j(x)$ in power series of $\mathcal{C}$,

$$
\begin{aligned}
\Lambda(x ; \mathcal{C}) & =\Lambda_{0}(x)+\Lambda_{1}(x) \mathcal{C}+\Lambda_{2}(x) \mathcal{C}^{2}+\cdots \\
j(x ; \mathcal{C}) & =j_{0}(x)+j_{1}(x) \mathcal{C}+j_{2}(x) \mathcal{C}^{2}+\cdots
\end{aligned}
$$

where $\Lambda_{n}(x)$ and $j_{n}(x), n=0,1,2, \cdots$, are functions of $x$ only. The expansion of $j(x ; \mathcal{C})$ follows directly from those of $e^{\lambda}$ and $e^{\nu}$. It is obvious that in the Newtonian limit $\mathcal{C} \rightarrow 0, j(x) \rightarrow j_{0}(x)=1$ and $\Lambda(x) \rightarrow \Lambda_{0}(x)=0$. On the other hand, integrating (3.1) with the regularity boundary condition of $\Lambda$ at $x=0$ leads to

$$
\frac{d \Lambda}{d x}=-\frac{1}{x^{4} j} \int_{0}^{x} 4 x^{\prime 3} \frac{d j}{d x^{\prime}}(\Lambda-1) d x^{\prime} .
$$

In consideration of the fact that $d j / d x=j_{1}^{\prime}(x) \mathcal{C}+j_{2}^{\prime}(x) \mathcal{C}^{2}+\cdots$, with $j_{n}^{\prime}(x) \equiv d j_{n} / d x$, we define $d \Lambda / d x$ as $\mathcal{C} \Psi$, where $\Psi$ is expandable in a regular power series of compactness $\mathcal{C}$ as:

$$
\Psi(x ; \mathcal{C})=\Psi_{0}(x)+\Psi_{1}(x) \mathcal{C}+\Psi_{2}(x) \mathcal{C}^{2}+\cdots,
$$


with $\Psi_{n}=d \Lambda_{n+1} / d x$ for $n \geq 0$. For example, from the expansions (3.2), (3.3) and (3.4) the first two functions $\Psi_{0}(x)$ and $\Psi_{1}(x)$ are given explicitly by the expressions:

$$
\begin{aligned}
& \Psi_{0}=-\frac{1}{x^{4} j_{0}} \int_{0}^{x} 4 x^{\prime 3} j_{1}^{\prime}\left(\Lambda_{0}-1\right) d x^{\prime} \\
& \Psi_{1}=\frac{j_{1}}{x^{4} j_{0}^{2}} \int_{0}^{x} 4 x^{\prime 3} j_{1}^{\prime}\left(\Lambda_{0}-1\right) d x^{\prime}-\frac{1}{x^{4} j_{0}} \int_{0}^{x} 4 x^{\prime 3}\left[j_{2}^{\prime}\left(\Lambda_{0}-1\right)+j_{1}^{\prime} \Lambda_{1}\right] d x^{\prime},
\end{aligned}
$$

while the others can similarly be found. It can also be seen that $\Psi_{n}$ is only related to $\Lambda_{i}$ 's with $0 \leq i \leq n$.

On the other hand, the continuity boundary conditions of $\Lambda$ and $d \Lambda / d x$ at the stellar surface imply that at $x=1$ $\Lambda_{n+1}=-\Lambda_{n+1}^{\prime} / 3$. Therefore, for $n \geq 0$

$$
\Lambda_{n+1}(x)=\int_{1}^{x} \Psi_{n}\left(x^{\prime}\right) d x^{\prime}-\Psi_{n}(1) / 3, n=0,1,2, \ldots .
$$

As mentioned above, $\Psi_{n}$ depends only on $\Lambda_{n}, \Lambda_{n-1}, \ldots, \Lambda_{0}$ and therefore $\Lambda_{n+1}$ can be determined from $\Lambda_{0}, \Lambda_{1}, \ldots, \Lambda_{n}$ in an iterative way. In particular, it can be shown from the recurrence relation exhibited in (3.8) that $\Psi_{n}$ and $\Lambda_{n}$ are $(2 n+1)$-th and $2 n$-th degree polynomials in $x$, respectively.

Carrying out the above-mentioned steps recursively, we find in turn the expansion of $\Lambda$,

$$
\Lambda(x ; \mathcal{C})=\frac{2}{5}\left(5-3 x^{2}\right) \mathcal{C}+\frac{1}{70}\left(21+126 x^{2}-99 x^{4}\right) \mathcal{C}^{2}+\cdots,
$$

which vanishes in the zero- $\mathcal{C}$ limit as expected. The expansion coefficients of $\Lambda(x ; \mathcal{C})$ up to $\mathcal{C}^{6}$-term are given in Table [II]. With the post-Minkowsian expansion for $\Lambda(x ; \mathcal{C})$, we can likewise find the expansion for the geometric factor $a$ :

$$
a(\mathcal{C})=a_{0}+a_{1} \mathcal{C}+a_{2} \mathcal{C}^{2}+\cdots,
$$

where the coefficients are given by $a_{n}=\hat{\Lambda}_{n+1} / 2$. The leading expansion (up to $\mathcal{C}^{6}$-term) for $a(\mathcal{C}$ ) is given by

$$
a(\mathcal{C})=\frac{2}{5}+\frac{12}{35} \mathcal{C}+\frac{212}{525} \mathcal{C}^{2}+\frac{632}{1155} \mathcal{C}^{3}+\frac{703744}{875875} \mathcal{C}^{4}+\frac{251264}{202125} \mathcal{C}^{5}+\frac{121542272}{60913125} \mathcal{C}^{6}+\cdots
$$

In the zero- $\mathcal{C}$ limit, $a=2 / 5$, which is the exact result of a Newtonian uniform sphere. The accuracy of (3.11) for relativistic incompressible stars is shown in Fig. 1, where the values of $a$ obtained from different schemes and the logarithm of the corresponding fractional deviations, $E \equiv \mid 1-$ (approximate value/exact value)|, are plotted against $\mathcal{C}$ in the top and bottom panels, respectively. The direct sum (DS) shown in (3.11), including terms up to $\mathcal{C}^{6}$, has an accuracy better than 0.01 until $\mathcal{C}$ is close to 0.4 .

The accuracy of the expansion in (3.11) can be further improved by constructing its Padé approximants (see, e.g., [34] for the theory and the construction of Padé approximants). In general, we can rewrite a $2 m$-th $(m=1,2,3, \cdots)$ order DS of a series $S$ such as (3.11) into a $(m, m)$ diagonal Padé approximant (denoted as $\mathrm{P}_{m}^{m}(S)$ hereafter), i.e., a rational function whose numerator and denominator are $m$-th degree polynomials in the expansion parameter $(\mathcal{C}$ in our case). For example, the $(2,2)$ Padé approximant of $a$ is given by

$$
\mathrm{P}_{2}^{2}(a)=\frac{\frac{2}{5}-\frac{1154648}{2116205} \mathcal{C}+\frac{24590348}{488843355} \mathcal{C}^{2}}{1-\frac{940102}{423241} \mathcal{C}+\frac{23746564}{23278255} \mathcal{C}^{2}}
$$

As shown in Fig. 1, for $\mathcal{C}>0.1$, the accuracy of $\mathrm{P}_{2}^{2}(a)$ is better than that of the DS containing terms up to $\mathcal{C}^{6}$, despite the fact that only terms up to $\mathcal{C}^{4}$ in the expansion (3.11) are required in the construction of $\mathrm{P}_{2}^{2}(a)$. As long as $\mathcal{C}<0.4$, Eq. (3.12) is accurate within $1 \%$. For $\mathcal{C}<0.1$, the accuracies of these two formulas are almost identical.

We note that Lattimer and Prakash [17] have previously obtained an empirical formula

$$
\begin{aligned}
a & =\frac{2}{5\left(1-0.87 \mathcal{C}-0.3 \mathcal{C}^{2}\right)} \\
& \approx \frac{2}{5}\left(1+0.87 \mathcal{C}+1.0569 \mathcal{C}^{2}+\cdots\right)
\end{aligned}
$$

by fitting the numerical values of the moment of inertia of incompressible stars. Comparing this empirical formula (termed as LP formula here) with the analytic result in (3.11), we see that the former is actually a good approximation 
of the latter at low compactness because, as shown by the second equality in (3.13), to order $\mathcal{C}^{2}$ these two expressions are quite close. In a sense, the LP formula is a $(0,2)$ Padé approximant for the geometric factor $a$. As shown in Fig. 11, in the low-compactness regime, the fractional deviation of the LP formula in (3.13) is greater than those of (3.11) and (3.12), reflecting the significance of higher order terms in (3.11). As $\mathcal{C}$ grows larger 0.22 , the errors arising from the LP formula and the DS (3.11) are comparable, though the latter still outperforms the former. In general, the $(2,2)$ Padé approximant in (3.12) has the best accuracy among the three approximations. This clearly demonstrates the advantage of Padé approximants.

\section{TIDAL DEFORMATION}

In this section, we calculate the tidal Love number (deformability) of incompressible stars and expand it in a series of compactness. In a binary system with two compact stars, the tidal field due to one compact star can induce quadrupole moments on its companion. This effect can be quantified with either the dimensionless tidal Love number $k_{2}$ or the tidal deformability $\lambda$, which relates the applied tidal fields $E_{i j}$ to the induced quadrupole moments $Q_{i j}$ through [1-4, 35]

$$
Q_{i j}=-\frac{2 k_{2} R^{5}}{3} E_{i j} \equiv-\lambda E_{i j}
$$

The dimensionless tidal deformability $\bar{\lambda}$ considered in the I-Love-Q relations is defined by $\bar{\lambda} \equiv \lambda / M^{5}=2 k_{2} /\left(3 \mathcal{C}^{5}\right)$.

The calculations of the Love number $k_{2}$ are given in 3, 36|. In the present paper we mainly follow the formulation established in [3]. With the conventions adopted in [37, 38], in electric tidal quadrupole deformation the metric perturbation $H=H_{0}=H_{2}$ satisfies the differential equation [3, 35]

$$
H^{\prime \prime}(r)+H^{\prime}(r)\left[\frac{2}{r}+e^{\lambda(r)}\left(\frac{2 m(r)}{r^{2}}+4 \pi r[p(r)-\rho(r)]\right)\right]+H(r) Q(r)=0,
$$

where, in general,

$$
Q(r)=4 \pi e^{\lambda(r)}\left[5 \rho(r)+9 p(r)+\frac{\rho(r)+p(r)}{c_{s}^{2}(r)}\right]-\frac{6 e^{\lambda(r)}}{r^{2}}-\left[\nu^{\prime}(r)\right]^{2} .
$$

For incompressible stars, the density $\rho(r)=\rho_{c}$, the sound speed $c_{s} \equiv \sqrt{d p / d \rho}$ tends to infinity and hence

$$
Q(r)=4 \pi e^{\lambda(r)}[5 \rho(r)+9 p(r)]-\frac{6 e^{\lambda(r)}}{r^{2}}-\left[\nu^{\prime}(r)\right]^{2} .
$$

After solving $H$, the Love number $k_{2}$ can be found from the following formula [3, 35]:

$$
\begin{aligned}
k_{2}\left(\mathcal{C}, y_{R}\right)= & \frac{8}{5} \mathcal{C}^{5}(1-2 \mathcal{C})^{2}\left[2 \mathcal{C}\left(y_{R}-1\right)+2\right]\left\{2 \mathcal{C}\left[4\left(y_{R}+1\right) \mathcal{C}^{4}+\left(6 y_{R}-4\right) \mathcal{C}^{3}+\left(26-22 y_{R}\right) \mathcal{C}^{2}+3\left(5 y_{R}-8\right)-3 y_{R}+6\right]\right. \\
& \left.+3(1-2 \mathcal{C})^{2}\left[2 \mathcal{C}\left(y_{R}-1\right)-y_{R}+2\right] \log (1-2 \mathcal{C})\right\}^{-1}
\end{aligned}
$$

where

$$
\begin{aligned}
y_{R} & \equiv\left(\frac{r}{H} \frac{d H}{d r}\right)_{r=R^{+}} \\
& =\left(\frac{r}{H} \frac{d H}{d r}-\frac{4 \pi R^{3} \rho}{M}\right)_{r=R^{-}} .
\end{aligned}
$$

It is readily shown from (4.2) that inside the star the logarithmic derivative of $H, y \equiv r H^{\prime}(r) / H(r)$, is governed by [35]

$$
r y^{\prime}(r)+y(r)^{2}+y(r) e^{\lambda(r)}\left\{1+4 \pi r^{2}[p(r)-\rho(r)]\right\}+r^{2} Q(r)=0,
$$

and the appropriate boundary condition at the center is $y(0)=2$. Once $y(r)$ and in turn $y_{R}$ are obtained, the Love number $k_{2}$ can be found from (4.5). 
Similar to the series expansion of $I$, we assume that $y$ can be expanded as a power series in $\mathcal{C}$

$$
y(x)=y_{0}(x)+y_{1}(x) \mathcal{C}+y_{2}(x) \mathcal{C}^{2}+y_{3}(x) \mathcal{C}^{3}+\cdots,
$$

where $y_{n}(x), n=0,1,2, \cdots$, are functions of $x$ only. We substitute (4.8) and the series expansions of $p, e^{\nu}$ and $e^{\lambda}$ (see (2.8), (2.9) and (2.10) ) into (4.7) and solve the resultant equation order by order, leading to a set of first-order ordinary differential equations:

$$
\begin{gathered}
x y_{0}^{\prime}(x)+y_{0}(x)^{2}+y_{0}(x)-6=0, \\
x y_{1}^{\prime}(x)+\left[1+2 y_{0}(x)\right] y_{1}(x)-x^{2} y_{0}(x)+3 x^{2}=0,
\end{gathered}
$$

and etc for $0 \leq x<1$. As $y_{n}(x)$ do not depend on $\mathcal{C}$, it follows directly from the boundary condition $y(0)=2$ that $y_{0}(0)=2$ and $y_{n}(0)=0$ for $n>0$. This leads to

$$
\begin{aligned}
& y_{0}(x)=2 ; \\
& y_{1}(x)=-\frac{1}{7} x^{2},
\end{aligned}
$$

and higher-order expansion can be obtained recursively. For reference the explicit expressions of $y_{n}$ up to $n=6$ are presented in Table IV]

Upon substitution of the so obtained $y_{n}$ into (4.8) and by (4.5) and (4.6), we can find the post-Minkowsian expansion for the Love number of incompressible stars:

$$
k_{2}=(1-2 \mathcal{C})^{2}\left(\frac{3}{4}-\frac{9}{7} \mathcal{C}+\frac{121}{294} \mathcal{C}^{2}-\frac{479}{11319} \mathcal{C}^{3}-\frac{196375}{1030029} \mathcal{C}^{4}-\frac{10670812}{21630609} \mathcal{C}^{5}-\frac{32621700682}{28314467181} \mathcal{C}^{6}+\cdots\right),
$$

and its $(2,2)$ Pade approximation

$$
\mathrm{P}_{2}^{2}\left(k_{2}\right)=(1-2 \mathcal{C})^{2} \frac{\frac{3}{4}+\frac{1213215}{2842294} \mathcal{C}-\frac{656811130}{328284357} \mathcal{C}^{2}}{1+\frac{3245062}{1421147} \mathcal{C}+\frac{76383026}{109428319} \mathcal{C}^{2}}
$$

In these two expressions for $k_{2}$, (4.13) and (4.14), we have deliberately kept the factor $(1-2 \mathcal{C})^{2}$ intact to manifest the black-hole limit as suggested in [3]. Figure 2 compares the exact numerical value of the Love number with the approximate values obtained from (4.13) and (4.14). In general, the agreement between the exact and the two approximate values is good, especially for small $\mathcal{C}$. As long as $\mathcal{C}<0.3$, the fractional deviations of the two approximations in (4.13) and (4.14) are less than 0.01, while the former is slightly smaller than latter. It should be noted that our main objective here is to derive the I-Love relation for realistic stars, whose compactnesses are in most cases less than 0.3. Therefore, both Eqs. (4.13) and (4.14) are accurate enough for the following discussion.

The expansion of the dimensionless tidal deformability of incompressible stars follows directly from (4.13),

$$
\bar{\lambda}=\frac{1}{2 \mathcal{C}^{5}}-\frac{20}{7 \mathcal{C}^{4}}+\frac{2515}{441 \mathcal{C}^{3}}-\frac{51550}{11319 \mathcal{C}^{2}}+\frac{3347350}{3090087 \mathcal{C}}+\frac{4326424}{64891827}+\frac{368458100}{9438155727} \mathcal{C}+\cdots .
$$

It is clearly seen from the above series that $\bar{\lambda}$ diverges as $1 /\left(2 \mathcal{C}^{5}\right)$ in the Newtonian limit. Besides, by inverting (4.15), we can express the compactness in terms of a power series in $\zeta \equiv(2 \bar{\lambda})^{-1 / 5}$ :

$$
\mathcal{C}=\zeta-\frac{8}{7} \zeta^{2}+\frac{430}{441} \zeta^{3}-\frac{24020}{33957} \zeta^{4}+\frac{1338940}{3090087} \zeta^{5}-\frac{207659912}{973377405} \zeta^{6}+\frac{130446932288}{1638194172615} \zeta^{7}+\cdots
$$

or equivalently

$$
\mathcal{C}=\frac{0.8706}{\bar{\lambda}^{1 / 5}}-\frac{0.8661}{\bar{\lambda}^{2 / 5}}+\frac{0.6433}{\bar{\lambda}^{3 / 5}}-\frac{0.4063}{\bar{\lambda}^{4 / 5}}+\frac{0.2167}{\bar{\lambda}}-\frac{0.09286}{\bar{\lambda}^{6 / 5}}+\frac{0.03017}{\bar{\lambda}^{7 / 5}}+\cdots .
$$

Eq. (4.16) or (4.17) is useful in the derivation of the I-Love relation. 


\section{I-LOVE RELATION FOR COMPACT STARS}

Yagi and Yunes [2] pointed out that there exists an almost EOS-independent relationship between the moment of inertia and the tidal Love number (deformability) of compact stars, which is universal to within $1 \%$. Their empirical fitting curve for the I-Love relation (referred to as YY formula here) is

$$
\ln \bar{I}=1.47+0.0817 \ln \bar{\lambda}+0.0149(\ln \bar{\lambda})^{2}+2.87 \times 10^{-4}(\ln \bar{\lambda})^{3}-3.64 \times 10^{-5}(\ln \bar{\lambda})^{4},
$$

with $\bar{I} \equiv I / M^{3}=a / \mathcal{C}^{2}$ being the dimensionless moment of inertia. The first (from top to bottom) panel of Fig. [3] shows a plot of $\log _{10} \bar{I}$ versus $\log _{10} \bar{\lambda}$ for realistic NSs with seven realistic EOSs (including APR 39], AU [40], BBB2 [41], FPS [42, 43], SLy4 [44], UU [40], WS [40, 43], one QS characterized by the MIT bag model [45, 46] and incompressible stars. It is seen that the YY formula is a good approximation as long as $\log _{10} \bar{\lambda}<4$. However, as can be observed from the second panel of Fig. 3, where the fractional deviation of the the YY formula (denoted by $E_{Y Y}$ ) is shown, the accuracy of the YY formula significantly worsens as $\log _{10} \bar{\lambda}$ grows beyond 4. In other words, the YY formula (5.1) does not cover the case of Newtonian stars.

Combining the series expansions (3.11) and (4.16) (or, equivalently, (4.17)), we can find an analytic I-Love relation for incompressible stars up to any desirable powers in $\bar{\lambda}^{-1 / 5}$. For example, the explicit expression for $\bar{I}$ up to $\zeta^{4}$ (or $\left.\bar{\lambda}^{-4 / 5}\right)$ is given by

$$
\begin{aligned}
\bar{I} & =\frac{2}{5 \zeta^{2}}+\frac{44}{35 \zeta}+\frac{17452}{11025}+\frac{31936}{33957} \zeta+\frac{21242792}{105343875} \zeta^{2}-\frac{990746384}{24334435125} \zeta^{3}-\frac{59041871509888}{1433419901038125} \zeta^{4}+\cdots, \\
& =\bar{\lambda}^{2 / 5}\left(0.5278+\frac{1.444}{\bar{\lambda}^{1 / 5}}+\frac{1.583}{\bar{\lambda}^{2 / 5}}+\frac{0.8187}{\bar{\lambda}^{3 / 5}}+\frac{0.1528}{\bar{\lambda}^{4 / 5}}-\frac{0.02686}{\bar{\lambda}}-\frac{0.02366}{\bar{\lambda}^{6 / 5}}+\cdots\right) .
\end{aligned}
$$

From the above DS formula, we can construct the $(m, m)$ diagonal Padé approximant $(m=1,2, \ldots)$ for $\bar{I} \zeta^{2}$ (or $\bar{I} / \bar{\lambda}^{2 / 5}$ ) by considering $\zeta$ (or $\bar{\lambda}^{-1 / 5}$ ) as the expansion parameter [34]. The formula for $\bar{I}$ resulting from the $(2,2)$ Padé approximant, which is constructed from the leading five terms of the DS in (5.2), is explicitly given by

$$
\begin{aligned}
\bar{I} & =\frac{\frac{2}{5}+\frac{44471656496}{50319113845} \zeta+\frac{2891441990432}{498592250655} \zeta^{2}}{\zeta^{2}\left(1-\frac{9393329026}{10063822769} \zeta+\frac{4965191579746}{11623715298195} \zeta^{2}\right)}, \\
& =\bar{\lambda}^{2 / 5}\left(\frac{0.5278+1.015 \bar{\lambda}^{-1 / 5}+0.5804 \bar{\lambda}^{-2 / 5}}{1-0.8126 \bar{\lambda}^{-1 / 5}+0.3237 \bar{\lambda}^{-2 / 5}}\right) .
\end{aligned}
$$

Although both (5.2) and (5.3) are intended for incompressible stars, as suggested in [25] and shown in Fig. 3, they are indeed good approximation for realistic NSs and QSs. In particular, the fractional deviations, $E_{D S}$ and $E_{P a d e}$, between $\bar{I}$ of various realistic NSs and QSs and the approximate values $\bar{I}$ of incompressible stars given by (5.2) and (5.3), respectively, are plotted against $\log _{10} \bar{\lambda}$ in the third and fourth panels of Fig. 3. It is clearly seen that $E_{D S}$ and $E_{P a d e}$ are generally comparable to each other. They are less than 0.02 for all realistic stars. For stars close to the maximum mass limit, $E_{D S}$ is still within 0.01 and smaller than $E_{\text {Pade }}$. On the other hand, the two deviations are very small towards the Newtonian limit. Hence, both (5.2) and (5.3) suffice to express the universal I-Love relation for realistic NSs, QSs and incompressible stars as well with good accuracy from the Newtonian limit to the maximum mass limit.

\section{CONCLUSION AND DISCUSSION}

In this paper, we derive from first principle the post-Minkowskian expansions for the moment of inertia and the Love number (or tidal deformability) of incompressible stars, expressing both physical quantities in terms of power series or Padé approximants of the compactness of the star. To our knowledge, such analytic expansions have not been available previously (see, e.g., the remark in [17]). The high accuracy of these formulas readily guarantees that they are useful in their own right. Due to the recursive nature of the expansion method employed in our derivation, the formulas for both quantities can be straightforwardly extended to higher orders should better precision for stars with large compactness is needed.

Furthermore, by eliminating the compactness from these two formulas, we obtain explicit equations, (5.2) and (5.3), directly connecting the moment of inertia and the Love number (or tidal deformability) of incompressible stars. Most importantly, Eqs. (5.2) and (5.3) are accurate enough to predict the universal behavior of realistic NSs and QSs to within $2 \%$ level (see Fig. 3). In fact, the agreement is much better than $1 \%$ as long as $\bar{\lambda}>10$ (i.e., not too dense stars). In comparison with the existing empirical formula, the present one has a broader range of applicability and 
is physically more transparent. For example, in the Newtonian limit, $\bar{\lambda} \rightarrow \infty$, it follows directly from (5.3) that $\bar{I}=2^{7 / 5} \bar{\lambda}^{2 / 5} / 5$, which agrees nicely with the Newtonian formula for incompressible stars obtained by Yagi and Yunes [1].

Our finding reported here also strongly supports the claim of Sham et al. 25] that the I-Love-Q universal relations of realistic NSs and QSs follow closely those of incompressible stars. As pointed out in [25], the physical origins of the I-Love-Q universal relations [1, 2] are the high stiffness of dense nuclear/quark matter and, in addition, the I-Love-Q relations are also insensitive to variation in stiffness around the incompressible limit. The good agreement between the theoretical I-Love formula and realistic data shown in Fig. 3] clearly lends support to the views proposed in [25].

To further justify theoretically the claim that the I-Love-Q relations are insensitive to variation in stiffness around the incompressible limit [25], explicit I-Love formulas for stars characterized by different stiffness (e.g. polytropic stars) should be sought. However, the derivation of the I-Love formula reported here crucially relies on the availability of the analytical solutions of the TOV equations for incompressible stars. To our knowledge, exact solutions for TOV equations of polytropic stars have not yet been derived. It will be helpful if such solutions can be worked out.

Lastly, a remark about the validity of (5.2) and (5.3) is in order. For a realistic NS near its low mass limit (typically, $\bar{\lambda}>10^{8}$ ) , a significant portion of its mass content is comprised of soft nuclear matter with polytropic index greater than unity. Hence, its behavior can no longer be approximated by the incompressible limit [25]. The accuracy of (5.2) and (5.3) is expected to worsen in this situation. However, NSs with such low compactness are not particularly interesting in astrophysics.

\section{Acknowledgments}

We thank L.-M. Lin, Y.H. Sham and H.K. Lau for helpful discussions. We are also grateful to Lin and Sham for providing us some numerical data presented here.

[1] K. Yagi and N. Yunes, Phys. Rev. D 88, 023009 (2013).

[2] K. Yagi and N. Yunes, Science 341, 365 (2013).

[3] T. Damour and A. Nagar, Phys. Rev. D 80, 084035 (2009).

[4] K. Yagi, Phys. Rev. D 89, 043011 (2014).

[5] A. Maselli, V. Cardoso, V. Ferrari, L. Gualtieri, and P. Pani, Phys. Rev. D 88, 023007 (2013).

[6] B. Haskell, R. Ciolfi, F. Pannarale, and L. Rezzolla, MNRAS 438, L71 (2014).

[7] G. Pappas and T. A. Apostolatos, Phys. Rev. Lett. 112, 121101 (2014).

[8] S. Chakrabarti, T. Delsate, N. Gürlebeck, and J. Steinhoff, Phys. Rev. Lett. 112, 201102 (2014).

[9] K. Yagi, K. Kyutoku, G. Pappas, N. Yunes, and T. A. Apostolatos, Phys. Rev. D 89, 124013 (2014).

[10] L. C. Stein, K. Yagi, and N. Yunes, Astrophys. J. 788, 15 (2014).

[11] E. E. Flanagan and T. Hinderer, Phys. Rev. D 77, 021502 (2008).

[12] T. Hinderer, Astrophys. J. 677, 1216 (2008).

[13] Y.-H. Sham, L.-M. Lin, and P. T. Leung, Astrophys. J. 781, 66 (2014).

[14] P. Pani and E. Berti, Phys. Rev. D 90, 024025 (2014).

[15] N. Andersson and K. D. Kokkotas, Phys. Rev. Lett 77, 4134 (1996).

[16] N. Andersson and K. D. Kokkotas, MNRAS 299, 1059 (1998).

[17] J. M. Lattimer and M. Prakash, Astrophys. J. 550, 426 (2001).

[18] J. M. Lattimer and B. F. Schutz, Astrophys. J. 629, 979 (2005).

[19] J. Lattimer and M. Prakash, Phys. Rep. 442, 109 (2007).

[20] F. Özel and D. Psaltis, Phys. Rev. D 80, 103003 (2009).

[21] W. C. G. Ho, N. Andersson, C. M. Espinoza, K. Glampedakis, B. Haskell, and C. O. Heinke, ArXiv e-prints (2013), 1303.3282.

[22] J. M. Lattimer and A. W. Steiner, Euro. Phys. J. A 50, 40 (2014).

[23] J. M. Lattimer and Y. Lim, Astrophys. J. 771, 51 (2013).

[24] K. Yagi, L. C. Stein, G. Pappas, N. Yunes, and T. A. Apostolatos, Phys. Rev. D 90, 063010 (2014).

[25] Y.-H. Sham, T. K. Chan, L.-M. Lin, and P. T. Leung, preprint, submitted to Astrophys. J. (2014).

[26] J. R. Oppenheimer and G. M. Volkoff, Phys. Rev. 55, 374 (1939).

[27] R. C. Tolman, Phys. Rev. 55, 364 (1939).

[28] R. C. Tolman, Relativity, Thermodynamics, and Cosmology (1934).

[29] J. B. Hartle, Gravity-An Introduction to Einstein's General Relativity (Addison Wesley, San Francisco, 2003).

[30] S. L. Shapiro and S. Teukolsky, Black Holes, White Dwarfs and Neutron Stars: The Physics of Compact Objects (Wiley, New York, 1983).

[31] J. B. Hartle, Astrophys. J. 150, 1005 (1967). 
[32] J. B. Hartle and K. S. Thorne, Astrophys. J. 153, 807 (1968).

[33] N. K. Glendenning, Compact Stars - Nuclear Physics, Particle Physics, and General Relativity (Springer, NY, 1997).

[34] G. Baker and P. Graves-Morris, Padé Approximants, Encyclopedia of Mathematics and Its Applications (Cambridge University Press, 1996).

[35] S. Postnikov, M. Prakash, and J. M. Lattimer, Phys. Rev. D 82, 024016 (2010).

[36] T. Hinderer, Astrophys. J. 677, 1216 (2008).

[37] J. R. Ipser and R. H. Price, Phys. Rev. D 43, 1768 (1991).

[38] L. Lindblom, G. Mendell, and J. R. Ipser, Phys. Rev. D. 56, 2118 (1997).

[39] A. Akmal, V. R. Pandharipande, and D. G. Ravenhall, Phys. Rev. C 58, 1804 (1998).

[40] R. B. Wiringa, V. Fiks, and A. Fabrocini, Phys. Rev. C 38, 1010 (1988).

[41] M. Baldo, I. Bombaci, and G. F. Burgio, Astron. Astrophys. 328, 274 (1997).

[42] V. R. Pandharipande and D. G. Ravenhall, Proc. NATO Advanced Research Workshop on nuclear matter and heavy ion collisions, Les Houches, (Plenum, New York, 1989), p. 103.

[43] C. P. Lorenz, D. G. Ravenhall, and C. J. Pethick, Phys. Rev. Lett. 70, 379 (1993).

[44] F. Douchin and P. Haensel, Astron. Astrophys. 380, 151 (2001).

[45] A. Chodos, R. L. Jaffe, K. Johnson, C. B. Thorne, and V. F. Weisskopf, Phys. Rev. D 9, 3471 (1974).

[46] E. Witten, Phys. Rev. D 30, 272 (1984). 
TABLE I: The coefficient of the $x^{j}$-term in the polynomials $\bar{p}_{n}(x) \equiv p_{n} / \rho_{c}$ for $0 \leq n \leq 6$. There is no odd power term and the coefficient vanishes if $j>2 n$.

\begin{tabular}{c|ccccccc} 
& $x^{0}$ & $x^{2}$ & $x^{4}$ & $x^{6}$ & $x^{8}$ & $x^{10}$ & $x^{12}$ \\
\hline $\bar{p}_{0}$ & 0 & 0 & 0 & 0 & 0 & 0 & 0 \\
$\bar{p}_{1}$ & $\frac{1}{2}$ & $-\frac{1}{2}$ & 0 & 0 & 0 & 0 & 0 \\
$\bar{p}_{2}$ & 1 & -1 & 0 & 0 & 0 & 0 & 0 \\
$\bar{p}_{3}$ & $\frac{17}{8}$ & $-\frac{19}{8}$ & $\frac{3}{8}$ & $-\frac{1}{8}$ & 0 & 0 & 0 \\
$\bar{p}_{4}$ & $\frac{37}{8}$ & $-\frac{23}{4}$ & $\frac{3}{2}$ & $-\frac{1}{4}$ & $-\frac{1}{8}$ & 0 & 0 \\
$\bar{p}_{5}$ & $\frac{163}{16}$ & $-\frac{223}{16}$ & $\frac{39}{8}$ & $-\frac{7}{8}$ & $-\frac{1}{16}$ & $-\frac{3}{16}$ & 0 \\
$\bar{p}_{6}$ & $\frac{723}{32}$ & $-\frac{539}{16}$ & $\frac{465}{32}$ & $-\frac{25}{8}$ & $\frac{5}{32}$ & $-\frac{3}{16}$ & $-\frac{9}{32}$
\end{tabular}

TABLE II: The coefficient of the $x^{j}$-term in the polynomials $\left(e^{\nu}\right)_{n}(x)$ for $0 \leq n \leq 6$. There is no odd power term and the coefficient vanishes if $j>2 n$.

\begin{tabular}{c|ccccccc} 
& $x^{0}$ & $x^{2}$ & $x^{4}$ & $x^{6}$ & $x^{8}$ & $x^{10}$ & $x^{12}$ \\
\hline$\left(e^{\nu}\right)_{0}$ & 1 & 0 & 0 & 0 & 0 & 0 & 0 \\
$\left(e^{\nu}\right)_{1}$ & -3 & 1 & 0 & 0 & 0 & 0 & 0 \\
$\left(e^{\nu}\right)_{2}$ & $\frac{3}{4}$ & $-\frac{3}{2}$ & $\frac{3}{4}$ & 0 & 0 & 0 & 0 \\
$\left(e^{\nu}\right)_{3}$ & $\frac{3}{4}$ & $-\frac{3}{4}$ & $-\frac{3}{4}$ & $\frac{3}{4}$ & 0 & 0 & 0 \\
$\left(e^{\nu}\right)_{4}$ & $\frac{15}{16}$ & $-\frac{3}{4}$ & $-\frac{3}{8}$ & $-\frac{3}{4}$ & $\frac{15}{16}$ & 0 & 0 \\
$\left(e^{\nu}\right)_{5}$ & $\frac{1171}{80}$ & $-\frac{15}{16}$ & $-\frac{3}{8}$ & $-\frac{3}{8}$ & $-\frac{15}{16}$ & $\frac{21}{16}$ & 0 \\
$\left(e^{\nu}\right)_{6}$ & $-\frac{2569}{240}$ & $\frac{961}{80}$ & $-\frac{15}{32}$ & $-\frac{3}{8}$ & $-\frac{15}{32}$ & $-\frac{21}{16}$ & $\frac{63}{32}$
\end{tabular}

TABLE III: The coefficient of the $x^{j}$-term in the polynomials $\Lambda_{n}(x)$ for $0 \leq n \leq 6$. There is no odd power term and the coefficient vanishes if $j>2 n$.

\begin{tabular}{c|ccccccc} 
& $x^{0}$ & $x^{2}$ & $x^{4}$ & $x^{6}$ & $x^{8}$ & $x^{10}$ & $x^{12}$ \\
\hline$\Lambda_{0}$ & 0 & 0 & 0 & 0 & 0 & 0 & 0 \\
$\Lambda_{1}$ & 2 & $-\frac{6}{5}$ & 0 & 0 & 0 & 0 & 0 \\
$\Lambda_{2}$ & $\frac{3}{10}$ & $\frac{9}{5}$ & $-\frac{99}{70}$ & 0 & 0 & 0 & 0 \\
$\Lambda_{3}$ & $\frac{2}{7}$ & $\frac{9}{25}$ & $\frac{72}{35}$ & $-\frac{199}{105}$ & 0 & 0 & 0 \\
$\Lambda_{4}$ & $\frac{2869}{8400}$ & $\frac{261}{700}$ & $\frac{549}{1400}$ & $\frac{11}{4}$ & $-\frac{3403}{1232}$ & 0 & 0 \\
$\Lambda_{5}$ & $\frac{1753}{3850}$ & $\frac{3439}{7000}$ & $\frac{927}{2450}$ & $\frac{373}{700}$ & $\frac{1541}{385}$ & $-\frac{851547}{200200}$ & 0 \\
$\Lambda_{6}$ & $\frac{54247603}{84084000}$ & $\frac{113063}{154000}$ & $\frac{88737}{196000}$ & $\frac{5169}{9800}$ & $\frac{9557}{12320}$ & $\frac{493299}{80080}$ & $-\frac{11684429}{1716000}$
\end{tabular}


TABLE IV: The coefficient of the $x^{j}$-term in the polynomials $y_{n}(x)$ for $0 \leq n \leq 6$. There is no odd power term and the coefficient vanishes if $j>2 n$.

\begin{tabular}{c|ccccccc} 
& $x^{0}$ & $x^{2}$ & $x^{4}$ & $x^{6}$ & $x^{8}$ & $x^{10}$ & $x^{12}$ \\
\hline$y_{0}$ & 2 & 0 & 0 & 0 & 0 & 0 & 0 \\
$y_{1}$ & 0 & $-\frac{1}{7}$ & 0 & 0 & 0 & 0 & 0 \\
$y_{2}$ & 0 & $-\frac{33}{14}$ & $\frac{599}{294}$ & 0 & 0 & 0 & 0 \\
$y_{3}$ & 0 & $-\frac{33}{7}$ & $\frac{50}{49}$ & $\frac{12046}{3773}$ & 0 & 0 & 0 \\
$y_{4}$ & 0 & $-\frac{561}{56}$ & $\frac{1643}{392}$ & $-\frac{18561}{30184}$ & $\frac{146821487}{24720696}$ & 0 & 0 \\
$y_{5}$ & 0 & $-\frac{1221}{56}$ & $\frac{634}{49}$ & $-\frac{519}{343}$ & $-\frac{468151}{2060058}$ & $\frac{209428151}{19227208}$ & 0 \\
$y_{6}$ & 0 & $-\frac{5379}{112}$ & $\frac{28475}{784}$ & $-\frac{3345}{616}$ & $\frac{10010095}{8240232}$ & $-\frac{85525955}{115363248}$ & $\frac{27526499425793}{1359094424688}$
\end{tabular}




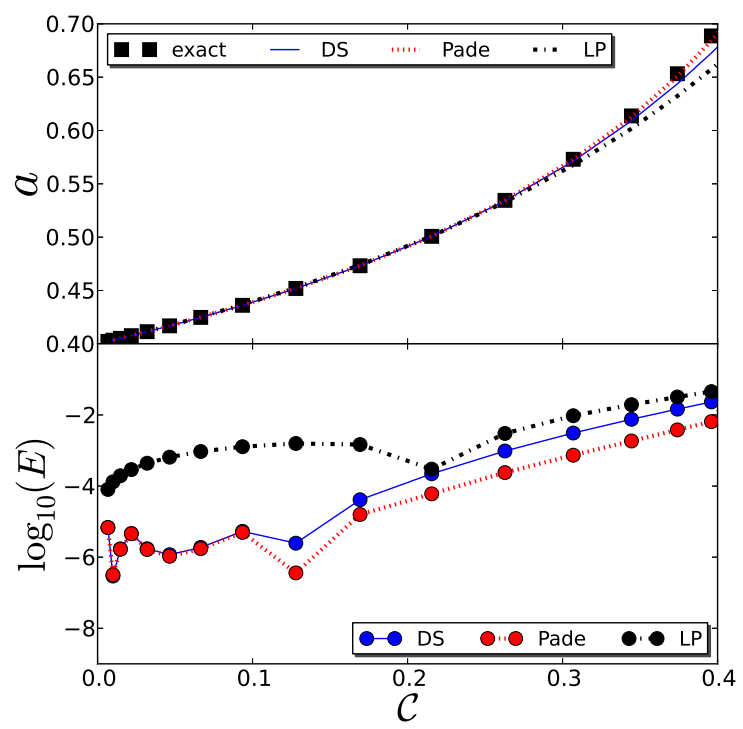

FIG. 1: Top panel: The geometric factor $a \equiv I /\left(M R^{2}\right)$ is plotted against compactness $\mathcal{C}$. The exact numerical value is denoted by solid squares. The approximate values of $a$ obtained from DS with terms up to $\mathcal{C}^{6}$ as shown in (3.11), (2,2) Padé approximant (3.12) and the LP empirical formula (3.13) are denoted by the continuous solid, dotted and dot-dashed curves, respectively. Bottom panel: The logarithm of the fractional deviations $(E)$ between the approximate values obtained from the above-mentioned formulas and the exact numerical value of $a$ are plotted against $\mathcal{C}$.

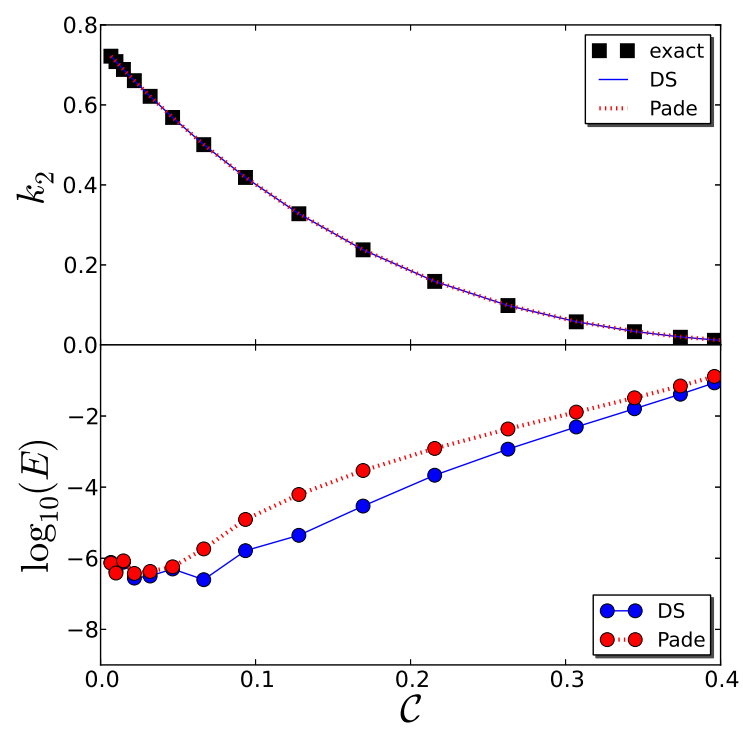

FIG. 2: Top panel: The tidal Love number $k_{2}$ is plotted against compactness $\mathcal{C}$. Sold squares represent exact numerical data. The continuous solid curve and dotted curve denote approximate values of $k_{2}$ obtained from DS in (4.13) with terms up to $\mathcal{C}^{6}$ and $(2,2)$ Padé approximant (4.14), respectively. Bottom panel: The logarithms of the fractional deviations for the two above-mentioned approximations from the exact value of $k_{2}$ are shown versus $\mathcal{C}$. 


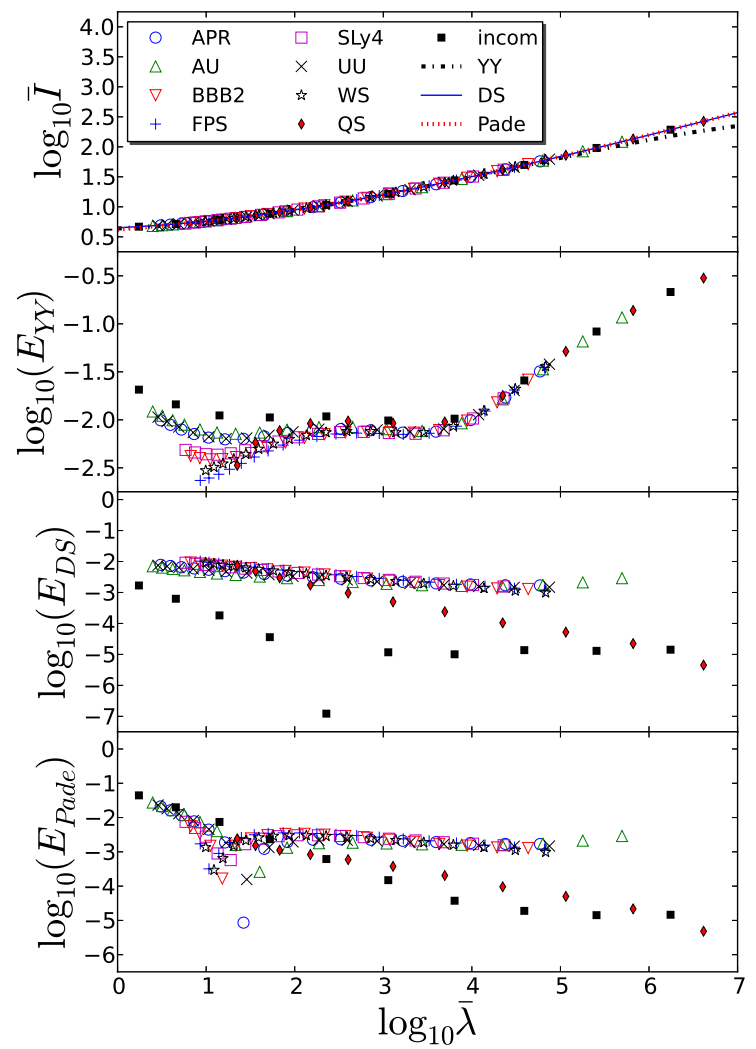

FIG. 3: First (from top to bottom) panel: $\log _{10} \bar{I}$ versus $\log _{10} \bar{\lambda}$. Exact data of NSs with seven realistic EOSs (including APR, AU, BBB2, FPS, SLy4, UU, WS), one QS and incompressible stars are shown. Besides, approximate values obtained from YY formula (5.1), sixth-order DS formula (5.2) and $(2,2)$ Padé approximant (5.3), respectively denoted by the dot-dashed, continuous and dotted curves, are also included. Second/Third/Fourth panel: The logarithm of the fractional deviation $E_{Y Y} / E_{D S} / E_{P a d e}$ between the YY/sixth-order DS/ 2,2$)$ Padé approximation of $\bar{I}$ and the exact value of $\bar{I}$ is shown against $\log _{10} \bar{\lambda}$. 